\title{
Blood-brain barrier pathology linked to epilepsy in Alzheimer disease
}

Epileptiform activity is a common occurrence in the brains of people with Alzheimer disease (AD), but the underlying mechanisms are poorly understood. A new study published in Science Translational Medicine identifies a possible EEG-based indicator of subclinical seizure activity in patients with $\mathrm{AD}$ and suggests a role for blood-brain barrier (BBB) dysfunction in AD-associated epilepsy.

A team led by Alon Friedman, who holds positions at Dalhousie University in Canada and Ben-Gurion University in Israel, examined EEG recordings from 39 individuals: 16 with $\mathrm{AD}, 12$ with mild cognitive impairment and 11 age-matched controls. The recordings revealed a specific pattern of transient slow brain activity, termed paroxysmal slow wave events (PSWEs), that was more frequent in the patients with $\mathrm{AD}$ than in the other two groups. The number of PSWEs per minute correlated with the degree of cognitive impairment.

In the next stage of the study, the researchers turned their attention to patients with epilepsy. EEG recordings from these individuals revealed the presence of interictal PSWEs. Most importantly, dynamic contrast-enhanced MRI (DCE-MRI) showed that this activity colocalized with areas of BBB disruption. A similar spatial relationship between PSWEs and $\mathrm{BBB}$ dysfunction was identified in three animal models: aged mice, the $5 \times$ FAD mouse model of $\mathrm{AD}$ and a rat model of status epilepticus.

To investigate the causal link between BBB disruption and PSWEs, Friedman and colleagues performed unilateral intraventricular injections of serum albumin into the brains of young rats. This intervention, which mimicked the effects of breaching

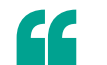

the investigators propose a mechanism whereby BBB disruption in AD and other neurological disorders induces PSWEs 5

the $\mathrm{BBB}$, was found to induce the development of PSWEs in the injected hemisphere.

On the basis of these multiple lines of evidence, the investigators propose a mechanism whereby $\mathrm{BBB}$ disruption in $\mathrm{AD}$ and other neurological disorders induces PSWEs, which provide an EEG signature of nonconvulsive seizure activity. As the team acknowledge, prospective trials that combine DCE-MRI scans with EEG recordings will be required to test this model.

"We have described a new approach to quantify transient shifts in cortical network activity using a simple, clinically applicable approach," concludes Friedman. "We further show that breaching the BBB is sufficient to introduce such changes in brain activity."

Heather Wood

ORIGINAL ARTICLE Milikovsky, D. Z. et al. Paroxysmal slow cortical activity in Alzheimer's disease is associated with blood-brain barrier dysfunction. Sci. Transl. Med. 11, eaaw8954 (2019) RELATED ARTICLE Sweeney, M. D. et al. Bloodbrain barrier breakdown in Alzheimer disease and other neurodegenerative disorders. Nat. Rev. Neurol. 14, 133-150 (2018)

Extracts from the brains of mice

\section{a-Synuclein strains induce distinct diseases in mice}

Different strains of misfolded $\alpha$-synuclein ( $\alpha$-syn) can cause clinically and pathologically distinct diseases in mice, according to a new study published in Nature Neuroscience. The findings highlight similarities between synucleinopathies and prion disease.

Synucleinopathies are neurodegenerative diseases that are characterized by the misfolding and aggregation of $\alpha$-syn in the brain. These diseases have different clinical and pathological features, although the reasons for the differences are unclear.

The misfolding of prion protein (PrP) also causes neurodegenerative disease. Misfolded PrP induces misfolding of healthy PrP, enabling the prion pathology to spread. Distinct conformational strains of PrP cause distinct diseases that can be transmitted between individuals by transfer of the diseases induced by the two strains of a-syn differed the misfolded protein. The new study, led by Joel Watts, aimed to establish whether different conformational forms of $\alpha$-syn can behave like strains of $\operatorname{PrP}$ and are responsible for different synucleinopathies.

The researchers generated two conformational strains of misfolded $\alpha$-syn, called 'S' and 'NS'. Both strains produced $\alpha$-syn pathology and neurological illness when injected into mice that expressed a mutant form of human $\alpha$-syn. Injection of properly folded $\alpha$-syn did not induce pathology in these mice. Importantly, the diseases induced by the two strains of $\alpha$-syn differed in their incubation periods, clinical signs and in the brain regions and cell types affected by $\alpha$-syn pathology. treated with the S or NS strains induced disease when injected into a second set of mice. Furthermore, when brain extracts from this second set of mice were injected into a third set of mice, the differences between the diseases caused by the N and NS strains were maintained.

"This observation means that strain-specific information is encoded within the structure of the $\alpha$-syn aggregates and can be transmitted from cell to cell and mouse to mouse in a 'prion-like' fashion," explains Watts.

The findings suggest that different synucleinopathies are caused by distinct strains of misfolded $\alpha$-syn, but further work is needed to confirm this hypothesis. "It is likely that the $\alpha$-syn strains we have uncovered so far will only partially mimic human synucleinopathies, so it will be important to continue to search for additional strains," concludes Watts.

Sarah Lemprière

ORIGINAL ARTICLE Lau, A. et al. $\alpha$-Synuclein strains target distinct brain regions and cell types. Nat. Neurosci. https://doi.org/10.1038/s41593019-0541-x (2019) 